Supporting Information

\title{
Rapid fabrication of poly(methyl methacrylate) devices for lab-on-a-chip applications using acetic acid and UV treatment
}

Kieu The Loan Trinh, ${ }^{1}$ Duc Anh Thai, ${ }^{2}$ Woo Ri Chae, ${ }^{3}$ Nae Yoon Lee ${ }^{*}, 2$

${ }^{1}$ Department of Industrial Environmental Engineering, Gachon University, 1342 Seongnam-daero, Sujeong-gu, Seongnam-si, Gyeonggi-do, 13120, Korea

2Department of BioNano Technology, Gachon University, 1342 Seongnam-daero, Sujeong-gu, Seongnam-si, Gyeonggi-do, 13120, Korea

${ }^{3}$ BioNano Research Institute, Gachon University, 1342 Seongnam-daero, Sujeonggu, Seongnam-si, Gyeonggi-do, 13120, Korea

*Corresponding Author: Tel: +82-31-750-8556, Fax: +82-31-750-8774, Email: nylee@gachon.ac.kr (N. Y. Lee) 
Table S1. Comparison of different solvent bonding methods

\begin{tabular}{|c|c|c|c|}
\hline Bonding process & Bond strength & Method description & Reference \\
\hline $\begin{array}{l}\text { - Acetic acid } 50 \% \\
\text { - UV irradiation ( } 30 \mathrm{~s} \text { ) } \\
\text { - Pressure (using clamp) } \\
\text { - Total process time: less than } 1 \mathrm{~min}\end{array}$ & $11.75 \mathrm{MPa}$ & $\begin{array}{l}\text { Advantages: } \\
\text { - Rapid and straightforward } \\
\text { - Low cost } \\
\text { - Non-toxic } \\
\text { - High bond strength } \\
\text { - Deformation-free and clogging-free } \\
\text { - Applicable for mass-production } \\
\text { Disadvantages: } \\
\text { - Requirement of well-ventilated facility to } \\
\text { eliminate acetic acid smell }\end{array}$ & This work \\
\hline $\begin{array}{l}\text { - Ethanol } 95 \% \\
\text { - Spin-coating and UV irradiation } \\
\text { - Total process time: around } 3 \mathrm{~min}\end{array}$ & $>1 \mathrm{MPa}$ & $\begin{array}{l}\text { Advantages: } \\
\text { - Simple and quick } \\
\text { - Low temperature } \\
\text { Disadvantages: } \\
\text { - Surface roughness affects the bonding } \\
\text { efficiency } \\
\text { - Solvent is flammable }\end{array}$ & [1] \\
\hline $\begin{array}{l}\text { - 1,2-dichloroethane and ethanol }(2: 8) \\
\text { - Room temperature } \\
\text { - Pressure (0.1 MPa) } \\
\text { - Total process time: around } 7 \mathrm{~min}\end{array}$ & $3.8 \mathrm{MPa}$ & $\begin{array}{l}\text { Advantages: } \\
\text { - Straightforward and simple } \\
\text { - Low temperature } \\
\text { Disadvantages: } \\
\text { - Solvent is highly flammable and volatile } \\
\text { - Requirement of pressure machine }\end{array}$ & [2] \\
\hline $\begin{array}{l}\text { - Chloroform and ethanol } \\
\text { - Heat at } 40^{\circ} \mathrm{C} \text { for } 10 \mathrm{~min} \\
\text { - Pressure (using quartz glass fixture) } \\
\text { - Total process time: around } 1 \mathrm{~h}\end{array}$ & $2.675 \mathrm{MPa}$ & $\begin{array}{l}\text { Advantages: } \\
\text { - Straightforward and simple } \\
\text { - Low pressure application } \\
\text { Disadvantages: } \\
\text { - Chloroform is toxic } \\
\text { - Time-consuming }\end{array}$ & [3] \\
\hline $\begin{array}{l}\text { - Acetonitrile } \\
\text { - Solvent exposure for } 8 \mathrm{~min} \\
\text { - Pressure (0.5 MPa) } \\
\text { - Total process time: around } 15 \mathrm{~min}\end{array}$ & Not mentioned & $\begin{array}{l}\text { Advantages: } \\
\text { - Simple and versatile } \\
\text { - Low cost } \\
\text { Disadvantages: } \\
\text { - Solvent is toxic and flammable } \\
\text { - Requirement of pressure machine }\end{array}$ & [4] \\
\hline $\begin{array}{l}\text { - Isopropanol } 100 \% \\
\text { - Ultrasonic }(8 \mathrm{~s}) \\
\text { - Pressure }(0.32 \mathrm{MPa}) \\
\text { - Total process time: less than } 2 \mathrm{~min}\end{array}$ & $2.25 \mathrm{MPa}$ & $\begin{array}{l}\text { Advantages: } \\
\text { - Quick } \\
\text { - Low temperature } \\
\text { Disadvantages: } \\
\text { - Solvent is highly flammable and volatile } \\
\text { - Requirement of pressure machine }\end{array}$ & [5] \\
\hline
\end{tabular}

\section{References:}

(1) Chen, P. C.; Duong, L. H. Novel solvent bonding method for thermoplastic microfluidic chips. Sens. Actuator B-Chem. 2016, 237, 556-562.

(2) Lin, C. H.; Chao, C. H.; Lan, C. W. Low azeotropic solvent for bonding of PMMA microfluidic devices. Sens. Actuator B-Chem. 2007, 121(2), 698-705.

(3) Zhang, H.; Liu, X.; Li, T.; Han, X. Miscible organic solvents soak bonding method use in a PMMA multilayer microfluidic device. Micromachines 2014, 5, 1416-1428.

(4) Sun, X.; Peeni, B. A.; Yang, W.; Becerril, H. A.; Woolley, A. T. Rapid prototyping of poly(methyl methacrylate) microfluidic systems using solvent imprinting and bonding. J. Chromatogr. A 2007, 1162, 162-166.

(5) Zhang, Z.; Luo, Y.; Wang, X.; Zheng, Y.; Zhang, Y.; Wang, L. A low temperature ultrasonic bonding method for PMMA microfluidic chips. Microsyst. Technol. 2010, 16, 533-541. 


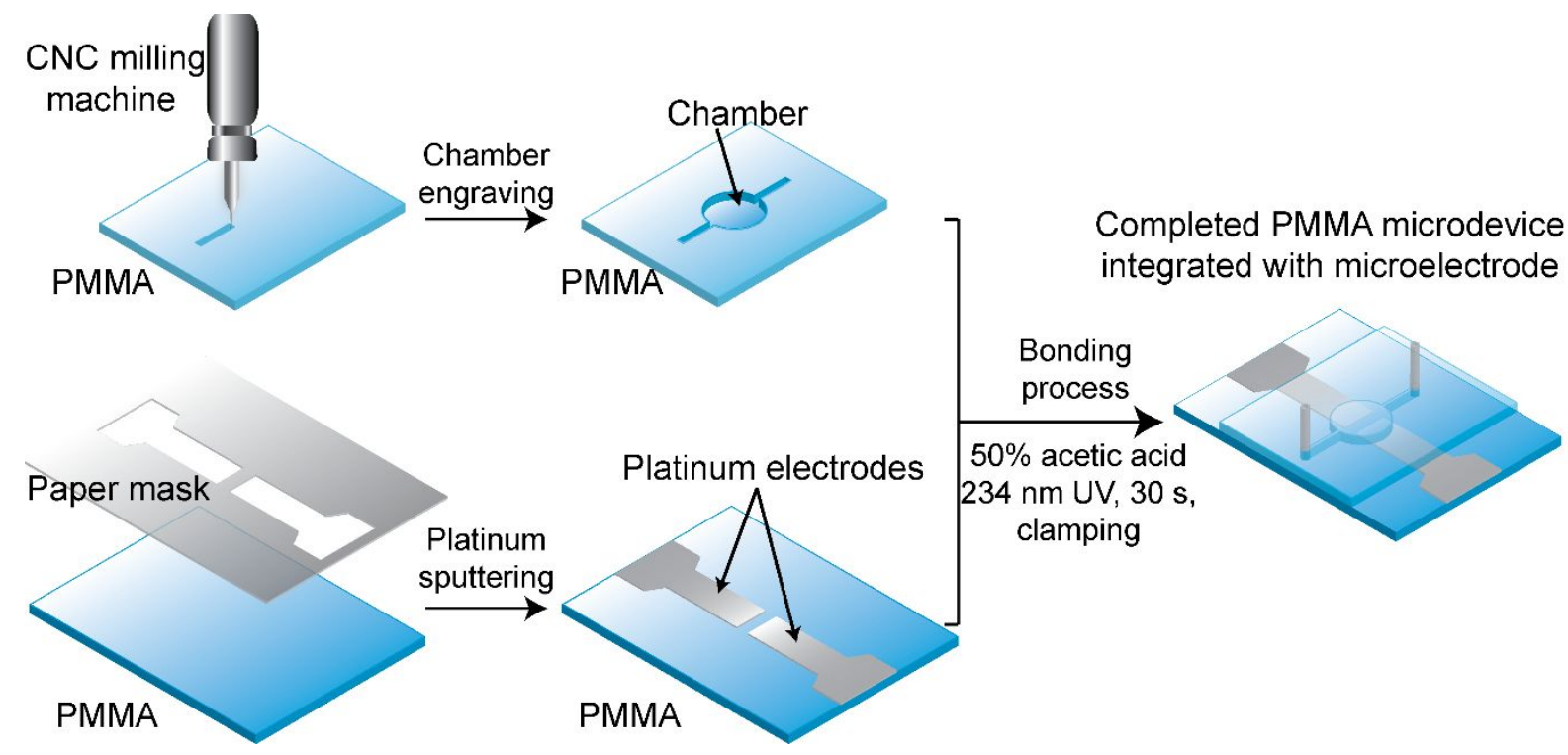

Figure S1. The overall schematic illustration of the fabrication of PMMA microdevice integrated with microelectrodes. 